\title{
Emerging treatments for premature ejaculation: focus on dapoxetine
}

\author{
Wayne JG Hellstrom \\ Chief, Section of Andrology, \\ Department of Urology, Tulane \\ University School of Medicine, New \\ Orleans, LA, USA
}

\begin{abstract}
Premature ejaculation (PE) is a common problem in men worldwide. It has a significant impact on affected men and their partners in terms of self-esteem, dissatisfaction with their sexual relationships, personal distress, and interpersonal difficulty. Psychological therapies may achieve short-term improvements, but there are limited data on the long-term success of these methods. Oral therapy with long-acting selective serotonin reuptake inhibitors (SSRIs) improves intravaginal ejaculatory latency time (IELT), but these agents are designed to be administered daily and may be associated with unwanted sexual side effects and withdrawal symptoms upon abrupt discontinuation. Dapoxetine is a short-acting SSRI that can be taken as needed (prn) by men with PE. It has been studied in five separate multicenter, randomized, double-blind, placebo-controlled trials involving more than 6000 men with PE. In four studies that evaluated IELT as an endpoint $(\mathrm{N}=4843)$, dapoxetine 30 and $60 \mathrm{mg}$ prn achieved statistically significant increases in IELT versus placebo. Dapoxetine also showed statistically significant improvements in perceived control over ejaculation, PE-related personal distress, and other patient-reported outcomes in all five trials. Dapoxetine treatment is generally well-tolerated, with low incidences of discontinuation syndrome, sexual dysfunction, and treatment-emergent mood symptoms. The most common adverse events with dapoxetine included nausea, diarrhea, headache, dizziness, and somnolence.
\end{abstract}

Keywords: dapoxetine, discontinuation syndrome, premature ejaculation, selective serotonin reuptake inhibitor

\section{Introduction}

Premature ejaculation (PE) is the most common form of male sexual dysfunction. ${ }^{1}$ Globally, between $20 \%$ and $40 \%$ of men, at some point in their lives, have reported symptoms of $\mathrm{PE}$ or a complaint of $\mathrm{PE},{ }^{1-3}$ which may be classified as lifelong or acquired. ${ }^{4}$ Lifelong PE is characterized by early ejaculation in the majority of intercourse attempts with nearly every partner from the first sexual encounter onwards, whereas acquired PE develops at some point in a man's life after he has previously experienced normal ejaculation and may be linked to urological or psychological problems. ${ }^{4}$ Some have also suggested that 2 additional forms of PE be considered, including natural-variable PE and premature-like ejaculatory dysfunction. Naturalvariable PE is characterized by early ejaculations that occur sporadically and should be regarded as a normal variation in sexual performance. ${ }^{4}$ Premature-like ejaculatory dysfunction is characterized by a false perception of PE even though these men have normal to very long ejaculation times. ${ }^{4}$

In clinical practice and for the purposes of clinical trials, the most commonly used definition of PE has been the Diagnostic and Statistical Manual of Mental Disorders, fourth edition, text revision (DSM-IV-TR), definition, which describes PE as "persistent or recurrent ejaculation with minimal sexual stimulation before, on, or shortly after penetration and before the person wishes it" that "causes marked distress or interpersonal 
difficulty" and "is not due exclusively to the direct effects of a substance." " More recently, the International Society for Sexual Medicine (ISSM) has more precisely defined lifelong PE as "a male sexual dysfunction characterized by ejaculation which always or nearly always occurs prior to or within about 1 minute of vaginal penetration; the inability to delay ejaculation on all or nearly all vaginal penetrations; and negative personal consequences, such as distress, bother, frustration and/or the avoidance of sexual intimacy."

The negative impact of PE on both the man and his female partner can be significant. The man may often feel ashamed and embarrassed at not being able to satisfy his partner; subsequently, low self-esteem, anxiety, and feelings of inferiority are common. ${ }^{7-10}$ Satisfaction with sexual intercourse and the sexual relationship may be reduced while personal distress and interpersonal difficulty may be increased in men with PE. ${ }^{8,10-12}$ Overall, many aspects of quality of life, including social functioning and emotional well-being, are significantly decreased. ${ }^{10}$ In two surveys of the female partners of men with PE, sexual satisfaction was "poor" or "very poor" in approximately $25 \%$ of respondents, personal distress related to PE was reported as at least "moderate" by approximately $50 \%$ of respondents, and interpersonal difficulty was at least "moderate" in up to $32 \%$ of respondents. ${ }^{11,13}$ The level of personal distress, perceived lack of control over ejaculation, satisfaction with sexual intercourse, and degree of interpersonal difficulty is comparable for men with PE and their female partners. ${ }^{12}$

Despite the high prevalence of PE, very few men seek treatment. ${ }^{14,15}$ In the Premature Ejaculation Prevalence and Attitudes (PEPA) survey of more than 12,000 men, only 9\% of men with self-reported PE sought help from a physician. ${ }^{2}$ Factors discouraging men from seeking treatment included doubts regarding the effectiveness of medication, concerns about becoming reliant on medication to perform sexually, and discomfort or embarrassment in discussing their PE. Of the men who had sought treatment for their PE, 92\% reported little or no improvement in their condition. Further, physicians rarely inquire about sexual health. In a separate international survey of 27,500 men and women aged 40 to 80 years, only $9 \%$ of respondents said they had been asked about their sexual health at routine visits within the previous 3 years, but more than $40 \%$ felt this should have occurred. ${ }^{15}$ These findings emphasize the need for an effective, well-tolerated, and acceptable treatment for PE.

\section{The physiology of ejaculation and the role of serotonin}

Ejaculation is a biphasic process consisting of emission, which involves the secretion of seminal fluid from the prostate and seminal vesicle, contraction of the smooth muscles of the seminal tract, and deposition of the sperm into the posterior urethra; and expulsion, which occurs when the semen is forced through the urethral meatus. ${ }^{16-18}$ Control mechanisms responsible for the inhibition of ejaculation descend from the supraspinal level and involve a number of regulatory neurotransmitters, the most widely studied of which is 5-hydroxytryptamine (5-HT), or serotonin. At least 3 serotonin receptor subtypes have been identified as having a role in ejaculation, including $5-\mathrm{HT}_{1 \mathrm{a}}, 5-\mathrm{HT}_{1 \mathrm{~b}}$, and $5 \mathrm{HT}_{2 \mathrm{c}}$. Although activation of the $5-\mathrm{HT}_{1 \mathrm{a}}$ receptor exhibits proejaculatory effects, activation of the $5-\mathrm{HT}_{1 \mathrm{~b}}$ and $5-\mathrm{HT}_{2 \mathrm{c}}$ receptors are involved in the process of delaying ejaculation. ${ }^{16,17}$ It has been hypothesized that altered levels of serotonin or serotonin receptor sensitivity in the ejaculatory modulating centers of the central nervous system are the pathophysiologic basis of ejaculatory disorders. ${ }^{17}$

\section{Current treatments for PE}

Historically, PE was considered a psychosomatic problem..$^{19,20}$ Therefore, behavioral, cognitive, and sex/relationship therapies have been a key component of PE management. Behavioral approaches have generally focused on the physical aspect of PE, including the "squeeze" technique as first described by Masters and Johnson in $1970^{21}$ and the "stop-start" method described by Semans in 1956. ${ }^{22}$ These involve engaging in sexual foreplay until just before the point of ejaculation and then either squeezing the head of the penis or stopping sexual activity until the urge to ejaculate subsides, at which point sexual activity may be resumed and the techniques repeated as necessary. Cognitive or sex therapy focuses on perceptions and feelings, improving communication between partners, increasing sexual skills and self-confidence, and reducing anxiety associated with sexual activity. ${ }^{19}$ Short-term improvement with these behavioral approaches has been observed; however, there are limited data concerning the efficacy of these methods in the long term. ${ }^{23-26}$

A number of pharmacologic therapies have been employed in the management of PE. Topical therapies for PE act by desensitizing the penis and do not alter the sensation of ejaculation. ${ }^{27} \mathrm{~A}$ topical cream containing local anesthetics lidocaine and prilocaine was effective in prolonging mean intravaginal ejaculatory latency time (IELT) by 6 to 8 minutes (vs 1-2 minutes with placebo; $\mathrm{p}<0.05$ ), but this must be applied at least 20 minutes (no longer than 45 minutes) prior to sexual contact. ${ }^{28,29}$ A novel aerosol formulation of lidocaine and prilocaine has also shown significant increases in IELT 
versus placebo (2.4- and 8-fold increases in two separate studies). ${ }^{30,31}$ Korean researchers have developed a topical cream (SS-cream) from natural products, which has local anesthetic properties and is applied to the penis 1 hour before sexual contact. ${ }^{27}$ In two randomized, double-blind, clinical trials, SS-cream (20-g dose) achieved a mean IELT of approximately 11 minutes, compared with 2.5 minutes for placebo. ${ }^{32,33}$

Such topical products can be applied on an as-needed (prn) basis and may be associated with fewer unwanted systemic adverse effects than oral therapies. However, side effects of these treatments may include loss of erection in men, a mild burning sensation within the vagina of the female partner, and numbness of the genitals in both partners. ${ }^{27,28,31}$ In addition, topical treatments can be messy and inconvenient to use because they must be applied and wiped off at specific times before sexual contact.

Selective serotonin reuptake inhibitors (SSRIs), commonly used in the treatment of depression, are often used to treat PE, based on the observation that delayed ejaculation is a frequent side effect of this drug class. In one prospective study $(\mathrm{N}=344)$ of patients being treated with SSRIs for psychiatric disorders, the incidence of delayed ejaculation ranged from $46 \%$ to $59 \% .^{34}$ In another study $(\mathrm{N}=1022)$, the incidence of delayed ejaculation ranged from $50 \%$ to $64 \% .{ }^{35}$ In both studies, paroxetine therapy was associated with the highest frequency of delayed ejaculation. In a third study of depressed patients $(\mathrm{N}=203)$, the incidence of abnormal ejaculation following paroxetine treatment was $24 \%$, compared with $12 \%$ for fluoxetine treatment. ${ }^{36}$

Results from several studies have shown that longacting SSRIs significantly prolong IELT in men with PE when administered with chronic daily dosing. For example, in a randomized, double-blind study $(\mathrm{N}=60)$, men with PE (defined as an IELT of $\leq 1$ minute) were randomized to receive 6 weeks of treatment with different long-acting SSRIs or placebo. Fluoxetine (20 mg/day), paroxetine (20 mg/day), and sertraline (50 mg/day) achieved statistically significant increases in mean IELT from baseline versus placebo (range of increases, $1.5-7$ minutes). ${ }^{37}$ In a separate, smaller study $(\mathrm{N}=34$, including 17 men and their female partners), treatment with paroxetine (20-40 mg/day) achieved a significant increase in median IELT from 0.5 minutes at baseline to 10 minutes after 6 weeks of treatment, compared with no change from baseline in median IELT with placebo therapy $(\mathrm{p}=0.002) .{ }^{38}$ Eight weeks of citalopram (20-60 mg/day) treatment in men with PE $(\mathrm{N}=26)$ has also been shown to significantly prolong IELT when compared with placebo (from 0.5 minutes at baseline to almost 5 minutes after 8 weeks; $\mathrm{p}<0.01) .{ }^{39}$ One analysis ${ }^{40}$ of two randomized, controlled, crossover studies $(\mathrm{N}=26$ and 42) compared IELT data among men who received paroxetine $20 \mathrm{mg} 3$ to 4 hours prior to sexual intercourse either alone or following initial daily treatment with paroxetine $10 \mathrm{mg}$. The results of this analysis found that IELT was significantly longer among men who were initially treated with paroxetine $10 \mathrm{mg}$ daily.

A key limitation of therapy for PE with currently available SSRIs is that in addition to delaying ejaculation, this class of drugs has been associated with a number of unwanted sexual side effects. Decreased libido (41\%-64\%, in men and women), anorgasmia (31\%-53\%, in men and women), and impotence/ erectile dysfunction (ED; $10 \%-41 \%$ ) have been observed following treatment with fluoxetine, paroxetine, fluvoxamine, sertraline, and citalopram. ${ }^{34,35}$ In addition, use of SSRIs may be associated with clinically significant effects on mood, such as reduced ability to cry, express feelings, or care about the feelings of others, in men with and without psychiatric conditions. ${ }^{41}$ Finally, abrupt cessation of SSRI therapy may also be associated with discontinuation syndrome, which includes a cluster of unpleasant physical and psychological symptoms including dizziness, nausea or vomiting, fatigue, headache, and unsteady gait. ${ }^{42}$ Discontinuation syndrome is considered to be more common with SSRIs that have shorter half-lives.

Other systemic treatments that have been evaluated for the management of PE include the phosphodiesterase-5 (PDE-5) inhibitors, currently licensed for the treatment of ED. The potential mechanism of action of PDE-5 inhibitors in the treatment of PE is unclear, but current proposals include a central effect via the nitric oxide/cyclic guanosine monophosphate pathway; peripheral effects on smooth muscle in the vas deferens, seminal vesicles, prostate, and urethra; the potential induction of peripheral analgesia; and prolongation of the duration of erection. ${ }^{43}$ Despite the success of PDE-5 inhibitors in treating ED, there have been limited data to suggest that these agents are effective in men without ED. For example, a single-dose, home-use study in young, healthy men found that sildenafil did not improve any aspect of erectile function or sexual performance in this population, although sildenafil was associated with a reduction in postejaculatory refractory time. ${ }^{44}$ A systematic review of clinical trials assessing sildenafil, tadalafil, and vardenafil concluded that changes in IELT with these agents are variable and difficult to interpret because of inconsistencies in study design. ${ }^{45}$ In an 8-week double-blind study, sildenafil prn increased mean IELT, but not to a significantly greater degree than placebo. ${ }^{46}$ Because of its mechanism of action, 
sildenafil may be associated with headache, flushing, dyspepsia, rhinitis, and visual disturbances in some subjects. The combination of an SSRI (paroxetine or fluoxetine) and sildenafil or tadalafil may improve IELT significantly more than the SSRI alone, but the incidence of side effects is increased relative to monotherapy with either of these agents. ${ }^{47-49}$ Treatment with PDE-5 inhibitors may be effective in men with comorbid ED and PE, and these two conditions commonly occur together. ${ }^{2,50}$ For example, McMahon and colleagues $^{45}$ suggested that combination therapy may be particularly effective for men with comorbid PE and ED; however, further study is needed in this area.

The oral opioid analgesic tramadol has also been assessed as a treatment for PE. Although the potential mechanism of action of tramadol in PE is not well understood, it may relate to the antinociceptive effects associated with $\mu$-opioid binding, which may decrease sensitivity, as well as the inhibition of norepinephrine and serotonin reuptake, which may delay ejaculation. ${ }^{51}$ Further investigation is needed to determine the precise effects of tramadol on $5 \mathrm{HT}_{1 \mathrm{a}}$ and $5 \mathrm{HT}_{2 \mathrm{c}}$. Two small clinical studies have evaluated the efficacy of tramadol in treating PE. In the first study $(\mathrm{N}=60)$, tramadol prn increased mean IELT from 1.2 minutes at baseline to 7.4 minutes at the end of 8 weeks, compared with a change from 1.1 to 2.0 minutes with placebo ( $p<0.0001$ ). In addition, $98 \%$ of subjects reported that they felt tramadol therapy was associated with a significant increase in their control over ejaculation. ${ }^{51}$ In the second study, an 8-week parallel-group study $(\mathrm{N}=64),{ }^{52}$ similar findings were reported.
In clinical practice, many of these treatment modalities are used in various combinations (eg, behavioral therapy and topical treatments); however, these combinations have rarely been rigorously evaluated in controlled clinical studies.

\section{Dapoxetine: an emerging pharmacologic treatment for PE}

Presently, no pharmacologic agent is approved by any regulatory agency specifically for the treatment of $\mathrm{PE}$, which may contribute to undertreatment of the condition. Dapoxetine is a novel short-acting SSRI under development for the treatment of PE. In preclinical models, dapoxetine has been statistically shown to significantly inhibit ejaculatory expulsion reflexes, acting at a supraspinal level. ${ }^{53,54}$ Similar to other SSRIs, dapoxetine exerts its effects primarily through the inhibition of the serotonin reuptake transporter, with minimal inhibitory activity at the norepinephrine and dopamine reuptake transporters. ${ }^{55}$ However, unlike long-acting SSRIs, which are typically administered in a chronic (daily) fashion and may take days or weeks to reach steady-state plasma concentrations, ${ }^{56}$ dapoxetine is a short-acting SSRI, which may be better suited to the prn treatment of PE. ${ }^{57}$

Compared with other agents currently in development for the treatment of PE, including new topical and other oral agents, the dapoxetine clinical development program is in the very late stages, as five phase III trials evaluating more than 6,000 subjects have been completed (Table 1).

Table I Design of the phase III clinical studies evaluating dapoxetine in premature ejaculation

\begin{tabular}{|c|c|c|c|c|}
\hline Country or region & Study population ${ }^{a}$ & Study duration & Study medication & Outcome measures \\
\hline $\begin{array}{l}\text { United States }{ }^{56} \text { ( } 2 \text { studies of identical } \\
\text { design) }\end{array}$ & 2614 & 12 weeks & $\begin{array}{l}\text { Dapoxetine } 30 \text { mg prn } \\
\text { Dapoxetine } 60 \text { mg prn } \\
\text { Placebo prn }\end{array}$ & $\begin{array}{l}\text { IELT } \\
\text { Control over ejaculation } \\
\text { Satisfaction with sexual } \\
\text { intercourse } \\
\text { CGIC }\end{array}$ \\
\hline Asia-Pacific ${ }^{57}$ & 1067 & 12 weeks & $\begin{array}{l}\text { Dapoxetine } 30 \text { mg prn } \\
\text { Dapoxetine } 60 \text { mg prn } \\
\text { Placebo prn }\end{array}$ & $\begin{array}{l}\text { IELT } \\
\text { PEP } \\
\text { CGIC }\end{array}$ \\
\hline International ${ }^{58}$ & 1162 & $\begin{array}{l}24 \text { weeks + I-week } \\
\text { randomized withdrawal }\end{array}$ & $\begin{array}{l}\text { Dapoxetine } 30 \text { mg prn } \\
\text { Dapoxetine } 60 \text { mg prn } \\
\text { Placebo prn }\end{array}$ & $\begin{array}{l}\text { IELT } \\
\text { PEP } \\
\text { CGIC } \\
\text { DESS }\end{array}$ \\
\hline $\begin{array}{l}\text { North America (Canada and the } \\
\text { United States) }\end{array}$ & 1238 & $\begin{array}{l}9 \text { weeks }+ \text { I-week } \\
\text { randomized withdrawal }\end{array}$ & $\begin{array}{l}\text { Double-dummy: } \\
\text { Dapoxetine } 60 \text { mg qd } \\
\text { Dapoxetine } 60 \text { mg prn } \\
\text { Placebo prn } \\
\text { Placebo qd }\end{array}$ & $\begin{array}{l}\text { PEP } \\
\text { CGIC } \\
\text { DESS }\end{array}$ \\
\hline
\end{tabular}

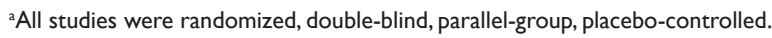

Abbreviations: prn, as needed; IELT, intravaginal ejaculatory latency time; CGIC, Clinical Global Impression of Change; PEP, Premature Ejaculation Profile; DESS, DiscontinuationEmergent Signs and Symptoms checklist; qd, once daily. 


\section{Pharmacokinetic profile of dapoxetine}

Dapoxetine $((+)-(\mathrm{S})-\mathrm{N}, \mathrm{N}-$ dimethyl-(a)-[2-(1naphthalenyloxy)ethyl]-benzenemethanamine hydrochloride) is a water-soluble powder with a molecular weight of 341.88 and a $\mathrm{pKa}$ of 8.6, which is charged at physiological $\mathrm{pH}^{58}$ and has a rapid pharmacokinetic profile. Dapoxetine is rapidly absorbed; after administration of single doses of dapoxetine 30 and $60 \mathrm{mg}$ in healthy male volunteers, peak plasma concentrations were observed 1.01 and 1.27 hours after administration, respectively. ${ }^{57}$ Elimination of dapoxetine is also rapid: the initial half-lives of dapoxetine 30 and $60 \mathrm{mg}$ are 1.3 and 1.4 hours, respectively. ${ }^{57}$ At 24 hours after administration, plasma concentrations of dapoxetine were below $4 \%$ of the peak for both doses. ${ }^{57}$ Multiple dosing of dapoxetine 30 and $60 \mathrm{mg}$ showed little change in this pharmacokinetic profile, with minimal apparent accumulation. ${ }^{57}$ This is in contrast to long-acting SSRIs. In 1 review of 5 SSRIs including fluoxetine, paroxetine, fluvoxamine, sertraline, and citalopram, half-lives were reported to range from 21 hours to 4 days. ${ }^{56}$ In addition, multiple dosing of long-acting SSRIs leads to significant accumulation; for example, chronic administration of paroxetine resulted in an 8-fold increase in plasma concentrations, ${ }^{59}$ while sertraline was found to result in a 2 -fold increase. ${ }^{60}$

\section{Clinical trial program for dapoxetine}

The clinical efficacy and safety of dapoxetine in men with PE has been extensively investigated in five separate phase III, randomized, double-blind, parallel-group, placebo-controlled clinical trials that enrolled more than 6000 subjects. All of these studies were similar in design. In all studies, men were 18 years or older with a DSM-IV-TR diagnosis of PE and were in a stable, monogamous, heterosexual relationship for at least 6 months ${ }^{61-64}$; these trials were conducted prior to the introduction of the ISSM criteria for PE. Four of these studies also required an IELT of 2 minutes or less at baseline for enrollment.

Efficacy measures across trials included IELT (included in four studies), the Premature Ejaculation Profile (PEP; included in three studies), and subject Clinical Global Impression of Change (CGIC; included in all 5 studies). The PEP is a validated instrument comprising 4 items related to PE (perceived control over ejaculation, personal distress related to ejaculation, satisfaction with sexual intercourse, and interpersonal difficulty related to ejaculation), each measured on five-point scales. ${ }^{11,65}$ The CGIC assesses change in overall condition, scored on a 7-point scale from -3 ("much worse") to 3 ("much better"). ${ }^{61}$ Additional outcome measures included the evaluation of sexual side effects and the potential of dapoxetine to cause anxiety, changes in mood or affect, or SSRI discontinuation syndrome following abrupt cessation. Anxiety-related symptoms were evaluated using the Hamilton Anxiety Scale (HAM-A), and moodand affect-related symptoms were measured using the Beck Depression Inventory II (BDI-II) and the MontgomeryÅsberg Depression Rating Scale (MADRS). Discontinuation syndrome was evaluated using the Discontinuation-Emergent Signs and Symptoms (DESS) checklist.

\section{Clinical outcomes with dapoxetine Intravaginal ejaculatory latency time}

Across the four trials that evaluated IELT, dapoxetine significantly improved IELT compared with baseline and placebo. In an integrated analysis of two US studies, ${ }^{61}$ mean IELT at baseline was approximately 0.9 minutes in all treatment groups. At the Week 12 endpoint, this increased to 1.75 minutes in the placebo group, 2.78 minutes in the dapoxetine $30-\mathrm{mg}$ group, and 3.32 minutes in the dapoxetine 60-mg group. Further results from this integrated analysis indicated that both doses of dapoxetine achieved significantly greater increases in mean IELT compared with placebo ( $\mathrm{p}<0.0001$ for both) and that dapoxetine $60 \mathrm{mg}$ showed a significantly greater increase in mean IELT than dapoxetine $30 \mathrm{mg}(\mathrm{p}=0.0007)$. Similar findings were observed in the subgroups of men with baseline IELT of less than 30 seconds (found in a post hoc analysis), less than 1 minute, or 1 to 2 minutes. The significant differences between dapoxetine and placebo were evident from the first dose and at all other time points (Weeks 4, 8, and 12). Based on CGIC data in this study, ${ }^{61}$ it was estimated that the minimum important change in IELT was about 1 minute, which was achieved by $22 \%, 47 \%$, and $51 \%$ of men receiving placebo, dapoxetine $30 \mathrm{mg}$, and dapoxetine $60 \mathrm{mg}$, respectively ( $\mathrm{p}<0.0001$ for dapoxetine vs placebo).

Similar findings were obtained in a 24-week international study conducted in 22 countries, primarily in Europe and South America. Mean IELT was approximately 0.9 minutes at baseline for all groups. By Week 12, IELT was significantly greater with dapoxetine $30 \mathrm{mg}$ (3.2 minutes) and $60 \mathrm{mg}$ (3.5 minutes) than with placebo (1.9 minutes; $\mathrm{p}<0.001$ for both); these results were maintained through the Week 24 endpoint. ${ }^{63}$ In a 12-week Asia-Pacific study, baseline mean IELT was approximately 1 minute in all treatment groups. At Week 12, the baseline mean IELT increased to 2.4 minutes with placebo, 3.9 minutes with dapoxetine $30 \mathrm{mg}$, and 4.2 minutes with dapoxetine $60 \mathrm{mg}$. The difference 
between both doses of dapoxetine and placebo was statistically significant $\left(\mathrm{p}<0.001\right.$ for both) ${ }^{62}$

\section{Patient-reported outcomes}

Across all trials of dapoxetine, all patient-reported measures of PE were significantly improved compared with both baseline and placebo. In the integrated analysis of two US studies, ${ }^{61}$ the percentage of subjects reporting "good" or "very good" perceived control over ejaculation improved from less than $0.3 \%$ of subjects across groups at baseline to $23.9 \%$ and $30.2 \%$ at 12 weeks with dapoxetine 30 and $60 \mathrm{mg}$, respectively (vs $8.7 \%$ with placebo; $\mathrm{p}<0.0001$ for both). Similar findings were observed for satisfaction with sexual intercourse. Although less than 21\% of subjects reported "good" or "very good" at baseline, $38.7 \%$ and $46.5 \%$ reported this level of satisfaction at 12 weeks with dapoxetine 30 and $60 \mathrm{mg}$, respectively (vs $24.6 \%$ with placebo; $\mathrm{p}<0.0001$ for both). In addition, the proportion of subjects rating their PE as "slightly better," "better," or "much better" on the CGIC at 12 weeks was significantly higher with both dapoxetine $30 \mathrm{mg}(58 \%)$ and $60 \mathrm{mg}(67 \%)$ than with placebo $(26 \%$; $\mathrm{p}<0.0001$ for both).

A North American trial ${ }^{64}$ conducted at multiple centers in Canada and the United States was designed primarily to evaluate the potential for SSRI withdrawal syndrome with prn and daily (qd) dosing regimens of dapoxetine $60 \mathrm{mg}$. The referenced study included a 9-week treatment phase followed by a 7-day withdrawal phase. Results from the treatment phase of this study focused on the dapoxetine 60-mg prn arm because dapoxetine is intended to be taken as needed. In this study, perceived control over ejaculation was rated as "good" or "very good" at baseline by no subjects in the placebo group and by only $0.2 \%$ of subjects in the dapoxetine 60 -mg prn group; however, at the study's end (Week 9) this increased to $20.4 \%$ with placebo and $39.7 \%$ with dapoxetine $60 \mathrm{mg}$ prn. ${ }^{64}$ Satisfaction with sexual intercourse was rated as "good" or "very good" by $9.5 \%$ and $7.9 \%$ of subjects in the placebo and dapoxetine 60-mg prn groups, respectively, at baseline. At study end, $54.7 \%$ of subjects receiving dapoxetine $60 \mathrm{mg}$ prn had achieved this status, compared with $34.0 \%$ of subjects receiving placebo. Although approximately 5\% of men in both groups reported "not at all" or "a little bit" for the level of personal distress related to ejaculation at baseline, $54.3 \%$ of those in the dapoxetine 60-mg prn group reported this level of distress at study endpoint (vs 35.3\% with placebo). Assessments of interpersonal difficulty related to ejaculation also improved greatly with dapoxetine. Although $43.0 \%$ and $40.9 \%$ of men reported "not at all" or "a little bit" for the level of interpersonal difficulty at baseline, this improved to $76.8 \%$ of subjects receiving dapoxetine by study endpoint (vs $64.2 \%$ with placebo). In addition, $41.3 \%$ of men in the dapoxetine 60-mg prn group rated their CGIC as "better" or "much better" compared with $20.8 \%$ of men in the placebo group $(\mathrm{p}<0.001)$.

Similar results were obtained in the international and Asia-Pacific trials. Preliminary data from the international trial showed that PRO measures, including perceived control over ejaculation and personal distress related to ejaculation, were significantly improved with dapoxetine 30 and $60 \mathrm{mg}$ versus placebo at Weeks 12 and 24 ( $\mathrm{p}<0.001$ for all). ${ }^{63}$ In addition, the percentage of men rating their PE as "slightly better," "better," or "much better" was $57.7 \%$ and $72.4 \%$ with dapoxetine 30 and $60 \mathrm{mg}$, respectively, compared with $32.0 \%$ with placebo (Figure 1) ${ }^{66}$ Preliminary results from the Asia-Pacific trial also showed significant improvements with dapoxetine compared with placebo for all PRO measures, including PEP items and CGIC.

\section{Benefits of dapoxetine for the female partner}

In addition to improving the condition in men with $\mathrm{PE}$, the female partners of men treated with dapoxetine also reported considerable benefits. In the integrated analysis of two US trials, ${ }^{61}$ the proportion of partners whose satisfaction with sexual intercourse at baseline was "fair," "good," or "very good" was $53 \%$ to $58 \%$ across the treatment groups. At the end of the study, this proportion remained unchanged in the placebo arm, whereas in the dapoxetine 30- and 60 -mg groups, the proportion increased to $72 \%$ and $78 \%$, respectively ( $\mathrm{p}<0.0001$ for both). Similar improvements were observed for the female partner's perception of the man's control over ejaculation. ${ }^{67}$

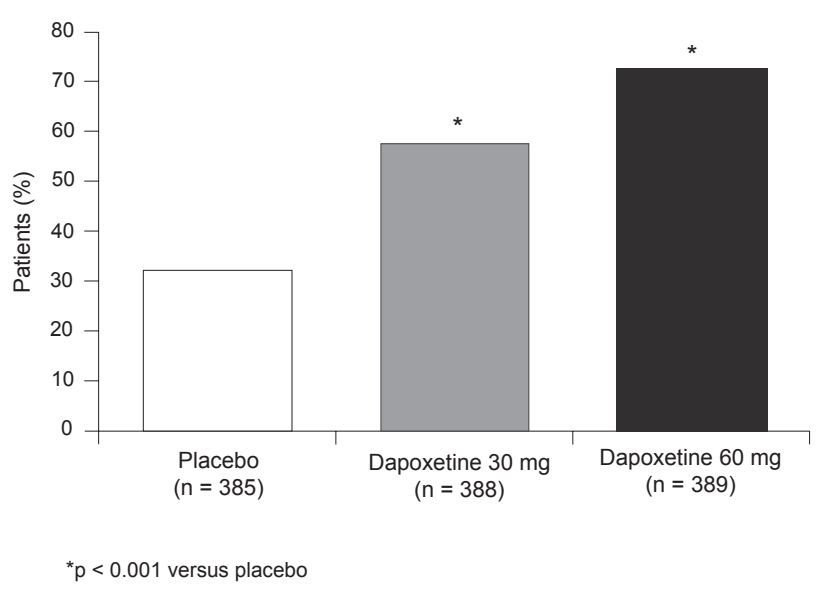

Figure I Patients achieving ratings of "slightly better," "better," or "much better" in Clinical Global Impression of Change in the international phase III clinical trial. 
In the international study, less than $5 \%$ of women across treatment groups reported their partner's control over ejaculation as "good" or "very good" at baseline. At the end of the 24-week treatment period, however, this increased to $25.0 \%$ and $32.3 \%$ for women whose partners were randomized to dapoxetine $30 \mathrm{mg}$ and $60 \mathrm{mg}$, respectively, compared with $14.4 \%$ of women whose partners were randomized to placebo ( $\mathrm{p}<0.001$ for both). ${ }^{68}$ Similarly, satisfaction with sexual intercourse was rated as "good" or "very good" by less than $16 \%$ of the partners at baseline. At study end, satisfaction with sexual intercourse increased significantly in both the dapoxetine 30-mg (33.8\%) and 60-mg (39.1\%) groups, compared with placebo (19.4\%; $\mathrm{p}<0.001$ for both).$^{68}$ Similar improvements were observed for partner-rated personal distress and interpersonal difficulty related to ejaculation. ${ }^{68}$

\section{Safety and tolerability with dapoxetine}

In the five phase III clinical trials, dapoxetine was generally well-tolerated and showed an acceptable safety profile. For example, the overall incidence of adverse events (AEs) in the Asia-Pacific study was $18 \%$ for placebo, $33 \%$ for dapoxetine $30 \mathrm{mg}$, and $50 \%$ for dapoxetine $60 \mathrm{mg} .{ }^{69} \mathrm{In}$ the international trial, the overall incidence of AEs was $38.4 \%, 56.2 \%$, and $68.1 \%$, respectively. ${ }^{63}$ Across trials, AEs were generally mild to moderate in severity.

The AE profile of dapoxetine exhibited some similarities with that reported for long-acting SSRIs, but also showed some important differences from these agents. The most frequently reported AEs following dapoxetine treatment, including nausea, diarrhea, headache, dizziness, and somnolence, are summarized in Table 2 for the integrated analysis of two US trials. ${ }^{61}$ These frequently-reported AEs occurred more often with dapoxetine $60 \mathrm{mg}$ than with dapoxetine $30 \mathrm{mg}$. A similar AE profile was observed in the international, North American, and Asia-Pacific trials. ${ }^{63,64,69}$
The incidence of severe or serious AEs was low $(<5 \%$ and $<1 \%$ of subjects, respectively) across trials. Less than $10 \%$ of subjects per treatment group in each study discontinued because of AEs, the most common of which was nausea ( $<4 \%$ in any treatment group). Discontinuation because of AEs occurred more often with dapoxetine $60 \mathrm{mg}$ than with dapoxetine 30 mg. ${ }^{61,63,64,69}$

The incidence of cardiovascular AEs in the US studies was low ( $<3 \%$ of subjects), and mean blood pressure and heart rate were similar with dapoxetine and placebo treatment. ${ }^{61}$ Syncope has been observed in the phase III trials. In the US studies, syncope was observed in 2 subjects receiving placebo, 3 receiving dapoxetine $30 \mathrm{mg}$, and 2 receiving dapoxetine $60 \mathrm{mg} .{ }^{61}$ Syncope was also experienced by 2 subjects receiving dapoxetine $60 \mathrm{mg}$ prn in the North American study. ${ }^{64}$ Syncope with dapoxetine appears to be vasovagal in nature and generally occurred following the first dose. In the clinical trial program, once subjects were educated to stay hydrated and recognize prodromal symptoms, there were no additional syncope events.

Dapoxetine has been associated with a low incidence of sexual side effects. During the 12-week double-blind treatment period in the integrated analysis of the two US studies, sexual dysfunction (ED, abnormal ejaculation, decreased libido, abnormal sexual function) was reported in $1.9 \%$ of subjects receiving placebo, compared with $4.5 \%$ of subjects receiving dapoxetine $30 \mathrm{mg}$ and $5.4 \%$ of subjects receiving dapoxetine $60 \mathrm{mg} .{ }^{61}$ In addition, in the 9-month open-label extension of these trials, during which all participants $(n=1774)$ received dapoxetine $60 \mathrm{mg}$, sexual side effects were reported by $2.6 \%$ of subjects. ${ }^{70}$ Similar low incidences of sexual AEs were reported for the 9-week treatment phase of the North American study (placebo: 2.4\%; dapoxetine $30 \mathrm{mg}$ : 3.3\%; dapoxetine $60 \mathrm{mg}$ : $3.6 \%$ ). ${ }^{71}$ As discussed previously, these findings are in contrast to the incidences of sexual dysfunction reported in clinical

Table 2 Summary of the most frequently-reported treatment-emergent adverse events in the integrated analysis of 2 US phase III studies

\begin{tabular}{|c|c|c|c|}
\hline Adverse event, $\%$ & Placebo $(n=870)^{a}$ & Dapoxetine $30 \mathrm{mg}(\mathrm{n}=874)^{\mathrm{a}}$ & Dapoxetine $60 \mathrm{mg}(\mathrm{n}=870)^{\mathrm{a}}$ \\
\hline Nausea & 1.9 & 8.7 & 20.1 \\
\hline Diarrhea & 1.4 & 3.9 & 6.8 \\
\hline Headache & 4.0 & 5.9 & 6.8 \\
\hline Dizziness & 0.8 & 3.0 & 6.2 \\
\hline Somnolence & 0.2 & 3.2 & 2.8 \\
\hline Serious adverse events & 0.9 & 0.3 & 0.6 \\
\hline
\end{tabular}

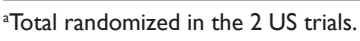

Adapted from The Lancet, 368, Pryor JL, Althof SE, Steidle C, et al. Efficacy and tolerability of dapoxetine in treatment of premature ejaculation: an integrated analysis of two double-blind, randomised-controlled trials. 929-937, 2006, ${ }^{61}$ with permission from Elsevier. 
studies of chronic long-acting SSRI therapy (up to $60 \%$ of subjects). ${ }^{34,35}$

The potential for SSRI discontinuation syndrome following abrupt withdrawal of dapoxetine was specifically evaluated in the international and North American trials, which included a 1-week randomized withdrawal from dapoxetine therapy. Discontinuation syndrome was defined as an increase of 4 points or more on the DESS checklist over the 7-day withdrawal period. ${ }^{72}$ In the international study, the incidence of discontinuation syndrome was $3.0 \%$ for subjects continuing to receive dapoxetine $30 \mathrm{mg}, 1.1 \%$ for those continuing on dapoxetine $60 \mathrm{mg}$, and $1.3 \%$ for those continuing on placebo. Of those subjects who switched from dapoxetine to placebo, no subjects previously taking dapoxetine $30 \mathrm{mg}$ and $3.3 \%$ of those previously taking dapoxetine $60 \mathrm{mg}$ met the criteria for discontinuation syndrome. ${ }^{72}$ In the North American study, upon withdrawal of dapoxetine 60 -mg qd treatment, $6.1 \%$ of subjects experienced insomnia (vs $2.4 \%$ of those remaining on dapoxetine therapy) and $4.8 \%$ (vs $1.2 \%$ of those remaining on dapoxetine therapy) experienced dizziness. ${ }^{72}$

The influence of dapoxetine on mood and affect, as measured by the BDI-II and the MADRS, was assessed during the initial 9-week double-blind treatment phase of the North American trial. ${ }^{71}$ Mean changes from baseline to study end for these rating scales were similar for dapoxetine $30 \mathrm{mg}$ and $60 \mathrm{mg}$ and placebo (BDI-II: $-1.4,-1.7,-1.2$, respectively; MADRS: $-0.1,-0.2,-0.1$, respectively). The incidence of depression-related AEs was less than $1 \%$ in all treatment groups.

In the North American and international studies, treatmentemergent anxiety was also assessed using the HAM-A. There were no significant differences between dapoxetine and placebo in changes from baseline in mean HAM-A total scores or individual item scores in either study. ${ }^{71,72}$ In the North American trial, the incidence of anxiety-related AEs was less than $2.5 \%$ across treatment groups. ${ }^{71}$

\section{Summary}

Premature ejaculation is a common sexual complaint among men of all ages, and it has a significant impact on men and their female partners. Current treatment options for PE have demonstrated limited long-term efficacy and/or undesirable side effects and have generally not been evaluated in large well-controlled clinical trials for the treatment of PE. Several new treatment options are in development for the treatment of PE, including oral and topical therapies.

Dapoxetine, a short-acting SSRI, is the most rigorously evaluated new agent being developed for the treatment of
PE. Dapoxetine has demonstrated efficacy and safety in five large, randomized, placebo-controlled phase III clinical trials. In addition to significantly prolonging IELT, subjects receiving dapoxetine have reported improvements in all other measures of PE evaluated, including perceived control over ejaculation, satisfaction with sexual intercourse, personal distress related to $\mathrm{PE}$, interpersonal difficulty related to $\mathrm{PE}$, and overall impression of change in PE severity. Dapoxetine is generally well-tolerated, with the most common side effect being nausea; in contrast to long-acting SSRIs, the incidence of undesirable sexual side effects, treatment-emergent anxiety, and discontinuation syndrome was low.

\section{Acknowledgments}

Editorial support was provided by Jason McDonough, PhD, of MedErgy.

\section{Disclosures}

The author has consulted for Johnson \& Johnson.

\section{References}

1. Althof SE. Prevalence, characteristics and implications of premature ejaculation/rapid ejaculation. J Urol. 2006;175(3 Pt 1):842-848.

2. Porst H, Montorsi F, Rosen RC, Gaynor L, Grupe S, Alexander J. The Premature Ejaculation Prevalence and Attitudes (PEPA) survey: prevalence, comorbidities, and professional help-seeking. Eur Urol. 2007;51(3):816-824.

3. Carson C, Gunn K. Premature ejaculation: definition and prevalence. Int J Impot Res. 2006;18(suppl 1):S5-S13.

4. Waldinger MD. Recent advances in the classification, neurobiology and treatment of premature ejaculation. Adv Psychosom Med. 2008;29:50-69.

5. American Psychiatric Association. Diagnostic and Statistical Manual of Mental Disorders, Fourth Edition, Text Revision. Washington, DC: American Psychiatric Association; 2000.

6. McMahon CG, Althof SE, Waldinger MD, et al. An evidence-based definition of lifelong premature ejaculation: report of the International Society for Sexual Medicine (ISSM) Ad Hoc Committee for the Definition of Premature Ejaculation. $J$ Sex Med. 2008;5(7):1590-1606.

7. Dunn KM, Croft PR, Hackett GI. Association of sexual problems with social, psychological, and physical problems in men and women: a cross sectional population survey. J Epidemiol Community Health. 1999;53(3):144-148.

8. Rowland D, Perelman M, Althof S, et al. Self-reported premature ejaculation and aspects of sexual functioning and satisfaction. $J$ Sex Med. 2004;1(2):225-232.

9. Sotomayor M. The burden of premature ejaculation: the patient perspective. J Sex Med. 2005;2(suppl 2):110-104.

10. Rowland DL, Patrick DL, Rothman M, Gagnon DD. The psychological burden of premature ejaculation. J Urol. 2007;177(3):1065-1070.

11. Patrick DL, Althof SE, Pryor JL, et al. Premature ejaculation: an observational study of men and their partners. J Sex Med. 2005;2(3):358-367.

12. Giuliano F, Patrick DL, Porst H, et al. Premature ejaculation: results from a five-country European observational study. Eur Urol. 2008;53(5):1048-1057.

13. Porst $\mathrm{H}$, Giuliano F, Patrick DL, et al. Impact of premature ejaculation (PE) on female partners: results from a 5-country European observational study [abstract]. Eur Urol Suppl. 2008;7(3):186. 
14. Carson CC, Glasser DB, Laumann EO, West SL, Rosen RC. Prevalence and correlates of premature ejaculation among men aged 40 years and older: a United States nationwide population-based study [abstract]. J Urol. 2003;169(4 suppl):321-322.

15. Moreira ED Jr, Brock G, Glasser DB, et al. Help-seeking behaviour for sexual problems: the Global Study of Sexual Attitudes and Behaviors. Int J Clin Pract. 2005;59(1):6-16.

16. Giuliano F. 5-hydroxytryptamine in premature ejaculation: opportunities for therapeutic intervention. Trends Neurosci. 2006; 30(2):79-84.

17. Wolters JP, Hellstrom WJ. Current concepts in ejaculatory dysfunction. Rev Urol. 2006;8(suppl 4):S18-S25.

18. Giuliano F, Hellstrom WJG. The pharmacological treatment of premature ejaculation. BJU Int. 2008;102(6):668-675.

19. Barnes T, Eardley I. Premature ejaculation: the scope of the problem. J Sex Marital Ther. 2007;33(2):151-170.

20. McMahon C. The etiology and management of premature ejaculation. Nat Clin Pract Urol. 2005;2(9):426-433.

21. Masters W, Johnson V. Human Sexual Inadequacy. Boston, MA: Little Brown and Co; 1970.

22. Semans J. Premature ejaculation: a new approach. $S$ Med J. 1956;49:353-358

23. De Amicis LA, Goldberg DC, LoPiccolo J, Friedman J, Davies L. Clinical follow-up of couples treated for sexual dysfunction. Arch Sex Behav. 1985;14(6):467-89.

24. Hawton K, Catalan J, Martin P, Fagg J. Long-term outcome of sex therapy. Behav Res Ther. 1986;24(6):665-675.

25. McCabe MP. Evaluation of a cognitive behavior therapy program for people with sexual dysfunction. J Sex Marital Ther. 2001;27(3):259-271.

26. Abdel-Hamid IA, El Naggar EA, El Gilany A-H. Assessment of as needed use of pharmacotherapy and the pause-squeeze technique in premature ejaculation. Int J Impot Res. 2001;13(1):41-45.

27. Morales A, Barada J, Wyllie MG. A review of the current status of topical treatments for premature ejaculation. BJU Int. 2007;100(3):493-501.

28. Atikeler MK, Gecit I, Senol FA. Optimum usage of prilocaine-lidocaine cream in premature ejaculation. Andrologia. 2002;34(6):356-359.

29. Busato W, Galindo CC. Topical anaesthetic use for treating premature ejaculation: a double-blind, randomized, placebo-controlled study. $B J U$ Int. 2004;93(7):1018-1021.

30. Henry R, Morales A. Topical lidocaine-prilocaine spray for the treatment of premature ejaculation: a proof of concept study. Int $J$ Impot Res. 2003;15(4):277-281.

31. Dinsmore WW, Hackett G, Goldmeier D, et al. Topical eutectic mixture for premature ejaculation (TEMPE): a novel aerosol-delivery form of lidocaine-prilocaine for treating premature ejaculation. BJU Int. 2006;99(2):369-375.

32. Choi HK, Xin ZC, Choi YD, Lee WH, Mah SY, Kim DK. Safety and efficacy study with various doses of SS-cream in patients with premature ejaculation in a double-blind, randomized, placebo controlled clinical study. Int J Impot Res. 1999;11(5):261-264.

33. Choi HK, Jung GW, Moon KH, et al. Clinical study of SScream in patients with lifelong premature ejaculation. Urology. 2000;55(2):257-261.

34. Montejo-González AL, Llorca G, Izquierdo JA, et al. SSRI-induced sexual dysfunction: fluoxetine, paroxetine, sertraline, and fluvoxamine in a prospective, multicenter, and descriptive clinical study of 344 patients. J Sex Marital Ther. 1997;23(3):176-194.

35. Montejo AL, Llorca G, Izquierdo JA, Rico-Villademoros F. Incidence of sexual dysfunction associated with antidepressant agents: a prospective multicenter study of 1022 outpatients. Spanish Working Group for the Study of Psychotropic-Related Sexual Dysfunction. J Clin Psychiatry. 2001;62(suppl 3):10-21.

36. Chouinard G, Saxena B, Bélanger M-C, et al. A Canadian multicenter, double-blind study of paroxetine and fluoxetine in major depressive disorder. J Affect Disord. 1999;54(1-2):39-48.
37. Waldinger MD, Hengeveld MW, Zwinderman AH, Olivier B. Effect of SSRI antidepressants on ejaculation: a double-blind, randomized, placebo-controlled study with fluoxetine, fluvoxamine, paroxetine, and sertraline. J Clin Psychopharmacol. 1998;18(4):274-281.

38. Waldinger MD, Hengeveld MW, Zwinderman AH. Paroxetine treatment of premature ejaculation: a double-blind, randomized, placebo-controlled study. Am J Psychiatry. 1994;151(9):1377-1379.

39. Atmaca M, Kuloglu M, Tezcan E, Semercioz A. The efficacy of citalopram in the treatment of premature ejaculation: a placebocontrolled study. Int J Impot Res. 2002;14(6):502-505.

40. McMahon CG, Touma K. Treatment of premature ejaculation with paroxetine hydrochloride as needed: 2 single-blind placebo controlled crossover studies. J Urol. 1999;161(6):1826-1830.

41. Opbroek A, Delgado PL, Laukes C, et al. Emotional blunting associated with SSRI-induced sexual dysfunction. Do SSRIs inhibit emotional responses? Int J Neuropsychopharmacol. 2002;5(2):147-151.

42. Black K, Shea C, Dursun S, Kutcher S. Selective serotonin reuptake inhibitor discontinuation syndrome: proposed diagnostic criteria. J Psychiatry Neurosci. 2000;25(3):255-261.

43. Chen J, Keren-Paz G, Bar-Yosef Y, Matzkin H. The role of phosphodiesterase type 5 inhibitors in the management of premature ejaculation: a critical analysis of basic science and clinical data. Eur Urol. 2007;52(5):1331-1339.

44. Mondaini N, Ponchietti R, Muir GH, et al. Sildenafil does not improve sexual function in men without erectile dysfunction but does reduce the postorgasmic refractory time. Int $J$ Impot Res. 2003;15(3):225-228.

45. McMahon CG, McMahon CN, Leow LJ, Winestock CG. Efficacy of type-5 phosphodiesterase inhibitors in the drug treatment of premature ejaculation: a systematic review. BJU Int. 2006;98(2):259-272.

46. McMahon CG, Stuckey BG, Andersen M, et al. Efficacy of sildenafil citrate (Viagra) in men with premature ejaculation. $J$ Sex Med. 2005;2(3):368-375.

47. Salonia A, Maga T, Colombo R, et al. A prospective study comparing paroxetine alone versus paroxetine plus sildenafil in patients with premature ejaculation. J Urol. 2002;168(6):2486-2489.

48. Hosseini MM, Yarmohammadi H. Effect of fluoxetine alone and in combination with sildenafil in patients with premature ejaculation. Urol Int. 2007;79(1):28-32.

49. Mattos RM, Marmo Lucon A, Srougi M. Tadalafil and fluoxetine in premature ejaculation: prospective, randomized, double-blind, placebo-controlled study. Urol Int. 2008;80(2):162-165.

50. Malavige LS, Jayaratne SD, Kathriarachchi ST, Sivayogan S, Fernando DJ, Levy JC. Erectile dysfunction among men with diabetes is strongly associated with premature ejaculation and reduced libido. $J$ Sex Med. 2008;5(9):2125-2134.

51. Salem EA, Wilson SK, Bissada NK, Delk JR, Hellstrom WJ, Cleves MA. Tramadol HCL has promise in on-demand use to treat premature ejaculation. $J$ Sex Med. 2008;5(1):188-193.

52. Safarinejad MR, Hosseini SY. Safety and efficacy of tramadol in the treatment of premature ejaculation: a double-blind, placebocontrolled, fixed-dose, randomized study. J Clin Psychopharmacol. 2006;26(1):27-31.

53. Clément P, Bernabé J, Gengo P, et al. Supraspinal site of action for the inhibition of ejaculatory reflex by dapoxetine. Eur Urol. 2007;51(3):825-832.

54. Giuliano F, Bernabe J, Gengo P, Alexandre L, Clement P. Effect of acute dapoxetine administration on the pudendal motoneuron reflex in anesthetized rats: comparison with paroxetine. $J$ Urol. 2007;177(1):386-389.

55. Gengo PJ, Giuliano F, McKenna K, et al. Monoaminergic transporter binding and inhibition profile of dapoxetine, a medication for the treatment of premature ejaculation [abstract]. $J$ Urol. 2005;173(4):239.

56. Hiemke C, Hartter S. Pharmacokinetics of selective serotonin reuptake inhibitors. Pharmacol Ther. 2000;85(1):11-28. 
57. Modi NB, Dresser MJ, Simon M, Lin D, Desai D, Gupta S. Single- and multiple-dose pharmacokinetics of dapoxetine hydrochloride, a novel agent for the treatment of premature ejaculation. J Clin Pharmacol. 2006;46(3):301-309.

58. Kendirci M, Salem E, Hellstrom WJ. Dapoxetine, a novel selective serotonin transport inhibitor for the treatment of premature ejaculation. Ther Clin Risk Manag. 2007;3(2):277-289.

59. PAXIL ${ }^{\circledR}$ (paroxetine hydrochloride) Tablets and Oral Suspension [package insert]. Research Triangle Park, NC: GlaxoSmithKline; 2007.

60. ZOLOFT ${ }^{\circledR}$ (sertraline hydrochloride) Tablets and Oral Concentrate [package insert]. New York, NY: Pfizer Inc; 2005.

61. Pryor JL, Althof SE, Steidle C, et al. Efficacy and tolerability of dapoxetine in treatment of premature ejaculation: an integrated analysis of two double-blind, randomised-controlled trials. Lancet. 2006;368(9539):929-937.

62. McMahon C, Park NC, Zhao Y, Rothman M, Rivas D. Treatment of premature ejaculation (PE) in the Asia-Pacific region: results from a phase III double-blind, parallel-group study of dapoxetine. Oral presentation at: 11th Biennial Meeting of the Asia Pacific Society for Sexual Medicine; October 6-10, 2007; Jeju, Korea. Abstract OS05_06.

63. Buvat J, Giuliano F, Tesfaye F, Rothman M, Rivas D. Efficacy and safety of dapoxetine for premature ejaculation (PE): results from a placebo-controlled, double-blind, randomized, parallel-group study in 22 countries [abstract]. Urology. 2007;70(3):15.

64. Kaufman JM, Rosen RC, Mudumbi RV, Tesfaye F, Hashmonay R, Rivas D. Treatment benefit of dapoxetine for premature ejaculation: results from a placebo-controlled phase III trial [published online ahead of print November 19, 2008]. BJU Int. doi:10.1111/ j.1464-410X.2008.08165.x.

65. Patrick DL, Giuliano F, Ho KF, Gagnon DD, McNulty P, Rothman M. The Premature Ejaculation Profile (PEP): validation of self-reported outcome measures for research and practice [published online ahead of print September 12, 2008]. BJU Int. doi:10.1111/j.1464 410X.2008.08041.x.
66. Buvat J, Giuliano F, Tesfaye F, Rothman M, Rivas D. Patient-reported treatment benefit of dapoxetine (DPX) for the treatment of premature ejaculation (PE) in 22 countries [abstract]. J Sex Med. 2008;5(suppl 1):30.

67. Brock G, Buvat J, Giuliano F, et al. Improvement in sexual satisfaction of female partners of men with premature ejaculation $(\mathrm{PE})$ treated with dapoxetine (DPX) [abstract]. J Urol. 2008;179(suppl 4):431-432.

68. Buvat J, Giuliano F, Tesfaye F, Rothman M, Rivas D. Improvement in sexual functioning of female partners of men with premature ejaculation (PE) treated with dapoxetine (DPX) [abstract]. Eur Urol Suppl. 2008;7(3):186.

69. Park NC, McMahon C, Tesfaye F, Zhao Y, Rothman M, Rivas D. Safety and tolerability of dapoxetine for the treatment of premature ejaculation in an Asia-Pacific population. Oral presentation at: 11th Biennial Meeting of the Asia Pacific Society for Sexual Medicine; October 6-10, 2007; Jeju, Korea. Abstract OS05_07.

70. Shabsigh R, Pryor JL, Tesfaye F. Dapoxetine for the treatment of premature ejaculation: low incidence of sexual side effects. In: Chen J, Arver S (chairpersons). P-05 Ejaculatory Disorders. J Sex Med. 2007;4(suppl 2):118.

71. Casey R, Tesfaye F, Mudumbi R, Rothman M, Rivas D. Effects of dapoxetine (DPX) for the treatment of premature ejaculation (PE) on the erectile function and scales of mood and affect [abstract]. Eur Urol Suppl. 2008;7(3):186.

72. Giuliano F, Levine SB, Buvat J, et al. Lack of withdrawal syndrome or effects on anxiety with dapoxetine (DPX) for the treatment of premature ejaculation (PE): results from 2 phase III trials [abstract]. Eur Urol Suppl. 2008;7(3):187. 